\title{
Antiretroviral Therapy Initiation and CD4 Progression over time among HIV Infected Adults in Central Africa
}

\author{
Hrishikesh Chakraborty ${ }^{1,2}$, Jamie E. Newman², Godfrey Woelk², Jennifer Hemingway-Foday², \\ Iriondo-PerezJeniffer ${ }^{2}$, Wilfred Akam ${ }^{3}$, Ashu Balimba ${ }^{4}$, Lucien Kalenga ${ }^{5}$, Marc Mbaya ${ }^{5}$, Henri Mukumbi ${ }^{5}$, \\ Théodore Niyongabo ${ }^{6}$, Brigitte MfangamMolu ${ }^{7}$, Robert Ryder $^{8}$ and Robin Huebner ${ }^{9}$ \\ ${ }^{1}$ Department of Epidemiology and Biostatistics, University of South Carolina, USA \\ ${ }^{2}$ Statistics and Epidemiology, RTI International, USA \\ ${ }^{3}$ Limbé Provincial Hospital, Limbé, Cameroon \\ ${ }^{4}$ Hôpital Militaire, Yaoundé, Cameroon \\ ${ }^{5}$ AMO-Congo, Democratic Republic of Congo \\ ${ }^{6}$ Centre Hospitalo-Universitaire de Kamenge, Bujumbura, Burundi \\ ${ }^{7}$ Hôpital Général de Yaoundé, Yaoundé, Cameroon \\ ${ }^{8}$ Department of Medicine, University of California San Diego, USA \\ ${ }^{9}$ National Institute of Allergy and Infectious Diseases, USA
}

\section{ABSTRACT}

An improved understanding of CD4 progression over time in resource-limited settings may aid in the discussion of when to initiate antiretroviral therapy (ART) and optimal timing for CD4 monitoring. We examined CD4 progression over time among 347 HIV-positive adults from $10 \mathrm{HIV}$ care and treatment programs in Burundi, Cameroon, and the Democratic Republic of Congo (DRC). We applied a mixed model using restricted maximum likelihood to describe CD4 cell count evolution over time following ART initiation and to evaluate the association between other baseline variables and CD4 response over time. Depending on the initial baseline CD4 count, increases in mean CD4 cell count ranged from $24-46$ cells $/ \mathrm{mm}^{3}$ from 3 to 6 months after treatment initiation, and from 80-117 from 6 to 18 months after treatment initiation. Females had higher CD4 count at the initiation of treatment than males. Patients with higher CD4 count at ART initiation achieved a higher CD4 count in the following months. We found that higher baseline CD4 cell counts predicted higher CD4 cell counts over time among the patients eligible for this analysis. We also observed increases in mean CD4 cell count from 3 to 18 months after treatment initiation.

Key Words: ART, CD4 Count, HIV, AIDS

\section{INTRODUCTION}

There were an estimated 33 million people living with HIV/AIDS worldwide in 2007 and by December 2008, 4 million people in low- and middle-income countries were receiving antiretroviral therapy (ART) [1]. ART is

Address for correspondence: Hrishikesh Chakraborty, DrPH

Department of Epidemiology and Biostatistics

Arnold School of Public Health

The University of South Carolina

800 Sumter Street, Suite 208-D

Columbia, SC 29208, Phone: 803-777-3170

Fax: 803-777-2524

E-mail: rishic@mailbox.sc.edu

DOI: $10.5530 /$ ijmedph.4.2011.2 an integral part of HIV prevention, care and support [2]. Benefits of ART include improved clinical status, increased CD4 cell count, and reduced viral load, thus extending the lives of people living with HIV/AIDS. Though strides have been made in treatment scale-up, much work remains to be done as more than 5 million people in low- and middle-income countries do not have access to much-needed ART [1]. Responses to ART in resource-limited settings approach those in high-income countries $[3,4,5]$, providing support for efforts to expand universal access. Two-thirds of people living with HIV/ AIDS reside in Sub-Saharan Africa, making it the most affected region in the world [1].

There is evidence that survival among HIV-infected adults is further improved when starting ART at CD4 
cell counts between 200 and 350 cells $/ \mathrm{mm}^{3}$ as compared to starting ART at less than 200 cells $/ \mathrm{mm}^{3}$, as is the standard of care in many resource-limited settings $[6,7,8,9]$. There is also evidence that early initiation of ART can decrease incident tuberculosis, a common and potentially lethal co-infection $[10,7,11]$. Early initiation of ART appears to also be more costeffective than delayed initiation [12, 13]. The World Health Organization (WHO) now recommends ART for HIV-infected adults and adolescents who have CD4 cell counts less than or equal to 350 cells $/ \mathrm{mm}^{3}$ [14]. However, support is building for starting ART even earlier. For example, Moore and Keruly's [15] findings support starting ART when CD4 counts are greater than 350 cells $/ \mathrm{mm}^{3}$. These investigators examined CD 4 counts over time stratified by CD 4 count at ART initiation $(<200,201-350,>350)$ and observed that by 6 years of treatment, the median CD 4 cell counts increased to 493 cells $/ \mathrm{mm}^{3}$ among patients in the United States with a baseline CD 4 count of $<200$, to 508 cells $/ \mathrm{mm}^{3}$ among those with a baseline CD4 count of 201-350, and to 829 cells $/ \mathrm{mm}^{3}$ among those with a baseline cell count $>350$. Even earlier initiation of ART (when CD4 cell counts are above 500 cells $/ \mathrm{mm}^{3}$ ) has been found to improve survival among HIV-positive adults in North America [16]. Additional inquiry is needed to ascertain the long-term benefits of early initiation of ART, particularly in regions most affected by the AIDS epidemic. An improved understanding of CD4 progression over time in resource-limited settings may aid in the continued discussion of optimal timing for initiating ART.

Countries hardest hit by the HIV/AIDS epidemic in SubSaharan Africa have navigated ART scale-up with limited laboratory monitoring. Resources have been balanced between increasing access to ART and increasing access to laboratory services. According to the recently reported results of the Development of Antiretroviral Therapy in Africa (DART) trial conducted in Uganda and Zimbabwe, ART can be delivered safely without routine CD4 monitoring [17]. However, differences in disease progression between the clinically driven monitoring group versus the laboratory and clinical monitoring group suggest a role for CD4 monitoring from the second year on to guide the switch to second-line therapy [17]. Increases in CD4 count were observed throughout the 5-year DART Trail for both study groups.

Determining when to initiate ART and optimal timing for CD4 monitoring is relevant for revising treatment guidelines, as well as planning for an increased need for antiretroviral and laboratory services. Due to gender differences reported from previous studies $[18,19,20]$, we assessed CD4 counts over time separately for males and females. The objective of this paper is to examine the CD4 progression over time among HIV-infected adults initiating ART and also to evaluate the association between other baseline variables and CD4 response over time in the central Africa region.

\section{METHODOLOGY}

Patients included in this analysis were participants in the International Epidemiologic Database to Evaluate AIDS (IeDEA) project, an initiative to collect and track clinical, laboratory, and epidemiologic data from HIV-infected individuals followed at globally distributed research units. The creation of this central database has enabled us to (1) address unique and evolving research questions in HIV/AIDS currently unanswerable by single cohorts, (2) increase the generalization of study results through the use of data from different settings and populations, and (3) provide a mechanism to more accurately define and monitor the HIV epidemic in an HIV-important and impoverished region.

The IeDEA Central Africa research project was reviewed by the Institutional Review Board (IRB) at the Kinshasa School of Public Health in DRC, the national ethics committees in Burundi and Cameroon, and the data coordinating center IRB at RTI International. The IRBs and ethics committees reviewing this research granted a waiver of consent for participants to be included in the IeDEA database.

The IeDEA Central Africa consortium collects adult data from $10 \mathrm{HIV}$ care and treatment centers in Burundi, Cameroon, and the Democratic Republic of Congo (DRC). The study began collecting data in the DRC in May 2007 and in Burundi and Cameroon in March 2008. As of April 2010, 15,116 HIV-positive adults had been enrolled in these three countries: DRC $(n=9,012)$, Cameroon ( $n=4,048)$, and Burundi $(n=2,056)$. The sample used in this analysis was selected from participants in the IeDEA database that had at least one CD4 count before starting ART and had at least one CD4 count during the next fourfollow-up visits. These criteria generated a final sample of $347 \mathrm{HIV}$-positive adults with the following distribution by country: DRC ( $\mathrm{n}=278)$, Cameroon $(\mathrm{n}=64)$, and Burundi $(\mathrm{n}=5)$. Patients that did not have CD4 counts $(n=14,769)$ were excluded.

Weevaluated how representative our patient subgroup was with patients enrolled in the IeDEA Central Africa database during the same time period (between March 1, 
2007, and July 15, 2007) by examining the 539 patients that had a CD4 count before starting ART and at least four follow-up visits. We compared demographic and clinical characteristics of our patient subgroup ( $\mathrm{n}=347$ ) with the characteristics of patients excluded from the analysis because they did not have a follow-up CD4 count $(n=192)$.

CD4 cell count was measured by the DRC National AIDS Reference Laboratory for the HIV care and treatment centers in DRC and by local reference laboratories for the centers in Cameroon and Burundi. Demographic characteristics and CD4 cell counts were obtained from the baseline survey and additional CD4 cell counts from subsequent follow-up visits. The baseline CD4 cell count was defined as the last CD4 count before ART initiation and was used as a categorical variable in this analysis $\left(<=100,101-200,201-300,>300\right.$ cells $\left./ \mathrm{mm}^{3}\right)$. The World Health Organization (WHO) Clinical Staging System for HIV for adults and adolescents was used to assess clinical stage [2]. Adherence was self-reported and defined as not missing antiretroviral drug (ARV) or other HIV-related medications for more than two consecutive days during the last month. Patients were asked to report medication adherence at each follow-up visit.

Chi-square $\left(\chi^{2}\right)$ tests were used to compare (1) baseline characteristics between the eligible patients for this analysis $(n=347)$ and the rest of the IeDEA cohort that was excluded due to lack of follow-up CD4 counts ( $n=192)$, and (2) characteristics of patients included in the study ( $\mathrm{n}=347$ ) among the different baseline CD4 count groups. Statistical significance was set at $\alpha=0.05$.

For describing CD4 cell count evolution over time following ART initiation, we applied a mixed-effects model with random intercept and slope using restricted maximum likelihood. The full model included fixed effects for baseline characteristics: baseline CD4 count group (<=100, 101-200, 201-300, >300 cells $/ \mathrm{mm}^{3}$ ); gender; health status as per physical exam (e.g., good, altered); age group (<=34; 35-39; 40-44; 45+); country (DRC/ Burundi and Cameroon); marital status (e.g., married, other); education (e.g., never/primary, secondary/ university); alcohol intake (i.e., yes, no); past history of TB (e.g., yes, no); skin condition (e.g., normal, lesion); and use of prophylactic cotrimoxazole (i.e., yes, no). Additionally, the model included two-way interactions between baseline CD4 count group and time, as well as baseline CD4 count group and the quadratic time term. All analyses were performed using SAS 9.1 for Windows (SAS Institute).

\section{RESULTS}

The final sample used in this analysis was 347 HIVpositiveadults. We excluded a substantial number of patients due to lack of CD4 counts ( $n=14,769)$. To determine how representative our patient subgroup was with patients enrolled in the IeDEA Central Africa database during the same time period, we examined the 539 patients that had a CD4 count before starting ART and at least 4 follow-up visits. The average time between the baseline CD4 measurement and the start of ARTvisit is 56 days (range: $1-1,269$ days) and the average time between the start of ART and the last follow-up visit is 587 days (range: $0-2,252$ days). As is presented in Table 1, patients in our analysis subgroup $(\mathrm{n}=347)$ did not differ from those excluded due to lack of follow-up CD4 count $(n=192)$ in terms of age, gender, WHO clinical stage, education, marital status, health status, skin condition, past history of TB, or receipt of cotrimoxazole prophylaxis. However, those in our patient subgroup were less likely to have consumed alcohol than those that were excluded due to lack of follow-up CD4 count (24\% vs. $43 \%, \mathrm{p}<0.0001)$. The difference of alcohol consumed is not significant in Cameroon (47.8\% vs. $40 \%$ ), but is significantly different in DRC and Burundi (40\% vs. $21 \%$, $\mathrm{p}<0.0001)$.

There were 283 patients included in this analysis from centers in DRC and Burundi (82\%) and 64 patients from centers in Cameroon (18\%). The majority of eligible patients in our subgroup were female $(72 \%)$, had secondary or university education $(71 \%)$, were in good health $(85 \%)$, and were married (which included monogamous and polygamous marriage) or were living together but not married (46\%). Most (56\%) of these patients were 40 years old or older. Half of the women were less than 40 years old as compared to $28 \%$ of men $(p<0.01)$. A total of $6(2 \%)$ women reported to be pregnant at the time of enrollment, and $19(8 \%)$ additional women were pregnant during follow-up. No statistically significant difference was observed between women and men for HIV clinical stage, health status, alcohol intake, past history of $\mathrm{TB}$, and prophylactic cotrimoxazole. A higher proportion of men $(65 \%)$ were married as compared to women $(39 \%)(p<0.001)$, while $68 \%$ of women have secondary/university education as compared to $81 \%$ of men $(p<0.001)$. Based on the information reported at their last follow-up visit, $333(96 \%)$ patients were taking ARVs. The main ART regimens were AZT $+3 \mathrm{TC}+\mathrm{NVP}$ (237 patients. $71 \%$ ), AZT+3TC+EFV (38 patients, $11 \%$ ), and $3 \mathrm{TC}+\mathrm{NVP}+\mathrm{d} 4 \mathrm{~T}-30$ (26 patients, $8 \%$ ), and the remaining 32 patients $(10 \%)$ were taking other ARTs. 
Table 1: Demographic and clinical characteristcs of patients used in the analysis $(n=347)$ versus patients that had a CD4 count before starting ART, had at least four follow-up visit, but had no follow-up CD4 count ( $n=192)$

\begin{tabular}{|c|c|c|c|}
\hline Demographics & Comparison group $\mathrm{n}(\%)$ & $\begin{array}{l}\text { Sample used in } \\
\text { the study } n(\%)\end{array}$ & p-value \\
\hline Age & 192 & 347 & 0.1492 \\
\hline$<=34$ & $66(34.4)$ & $93(26.8)$ & \\
\hline $35-39$ & $38(19.8)$ & $60(17.3)$ & \\
\hline $40-45$ & $34(17.7)$ & $79(22.8)$ & \\
\hline$>=45$ & $54(28.1)$ & 115(33.1) & \\
\hline Sex & 192 & 347 & 0.4534 \\
\hline Male & $49(25.5)$ & $99(28.5)$ & \\
\hline Female & $143(74.5)$ & $248(71.5)$ & \\
\hline HIV Stage & 192 & 346 & 0.4182 \\
\hline I & $26(13.5)$ & $34(9.8)$ & \\
\hline II & $51(26.6)$ & $98(28.3)$ & \\
\hline $\mathrm{III} / \mathrm{IV}$ & $115(59.9)$ & $214(61.8)$ & \\
\hline Education & 192 & 345 & 0.1362 \\
\hline Never/Primary & $67(34.9)$ & $99(28.7)$ & \\
\hline Secondary/University & $125(65.1)$ & 246 & \\
\hline Matital Status & 183 & 333 & 0.2654 \\
\hline Marrieda/Living Together & $94(51.4)$ & 154(46.2) & \\
\hline Other & $89(48.6)$ & $179(53.8)$ & \\
\hline Health Status & 192 & 347 & 0.7717 \\
\hline Good & $165(85.9)$ & $295(85.0)$ & \\
\hline Altered & $27(14.1)$ & $52(15.0)$ & \\
\hline Frequency of Alcohol intake & 192 & 347 & $<.0001^{*}$ \\
\hline Never & $110(57.3)$ & $263(75.8)$ & \\
\hline Daily/Weekly/Monthly & $82(42.7)$ & $84(24.2)$ & \\
\hline Skin Condition & 187 & 331 & 0.1600 \\
\hline Normal & $161(86.1)$ & 269(81.3) & \\
\hline Lesion & $26(13.9)$ & $62(18.7)$ & \\
\hline Past history of TB & 192 & 347 & 0.2026 \\
\hline Yes & $44(22.9)$ & $97(28.0)$ & \\
\hline No & $148(77.1)$ & $250(72.0)$ & \\
\hline Prophylactic cotrimoxazole & 191 & 347 & 0.3983 \\
\hline Yes & $131(68.6)$ & $250(72.0)$ & \\
\hline No & $60(31.4)$ & $97(28.0)$ & \\
\hline
\end{tabular}

A total $18 \%$ of males and $22 \%$ of females provided at least three follow-up observations; $26 \%$ of males and $31 \%$ females provided at least two follow-up observations; and $56 \%$ of males and $47 \%$ females provided at least one follow-up observation and were included in the analysis.

Out of 347 patients, only $59(17 \%)$ reported missing their medication at least once for more than two consecutive days during the follow-up period. There were 5,004 follow-up visits for these 347 patients, and in only 80 of these visits $(1.6 \%)$ didpatients report not being adherent to ART. There was no significant difference between women and men in self-reported adherence to treatment.

Among the baseline CD4 count $<=100$ group, 51\% provided at least one follow-up observation, 30\% provided at least two follow-up observations, and 19\% provided at least three follow-up observations. Among the baseline
CD4 count 101-200 group, 53\% provided at least one follow-up observation, $28 \%$ provided at least two follow-up observations, and 19\% provided at least three follow-up observations. Among the baseline CD4 count 201-300 group, $41 \%$ provided at least one follow-up observation, $33 \%$ provided at least twofollow-up observations, and $26 \%$ provided at least three follow-up observations. Among the baseline CwD4 count $>300$ group, 53\% provided at least one follow-up observation, $27 \%$ provided at least two follow-up observations, and $20 \%$ provided at least three follow-up observations.

Table 2 presents patient characteristics by baseline CD 4 count groups. There were no significant differences among CD4 count groups, except for health status. Higher CD4 counts were associated with better health $(p<0.001)$. Figure 1 illustrates the CD4 changes over time for each of the baseline CD 4 count groups. The data suggest a linear 


\begin{tabular}{|c|c|c|c|c|c|}
\hline \multirow[b]{2}{*}{ Demographics } & \multicolumn{4}{|c|}{ Baseline CD4 count group } & \multirow[b]{2}{*}{ p-value } \\
\hline & $\begin{array}{l}<=100 \text { cell/ } \\
\text { mm3 } n(\%)\end{array}$ & $\begin{array}{c}101-200 \text { cell/ } \\
\text { mm3 n (\%) }\end{array}$ & $\begin{array}{c}201-300 \text { cell/ } \\
\text { mm3 n (\%) }\end{array}$ & $\begin{array}{l}>300 \text { cell/ } \\
\text { mm3 } \mathrm{n}(\%)\end{array}$ & \\
\hline Age & 88 & 126 & 78 & 55 & 0.074 \\
\hline$<=34$ & $27(30.7)$ & $32(25.4)$ & $22(28.2)$ & $12(21.8)$ & \\
\hline $35-39$ & $17(19.3)$ & $14(11.1)$ & $14(17.9)$ & $15(27.3)$ & \\
\hline $40-45$ & $23(26.1)$ & $32(25.4)$ & $18(23.1)$ & $6(10.9)$ & \\
\hline$>=45$ & $21(23.9)$ & $48(38.1)$ & $24(30.8)$ & $22(40.0)$ & \\
\hline Sex & 88 & 126 & 78 & 55 & 0.215 \\
\hline Male & $26(29.5)$ & $40(31.7)$ & $15(19.2)$ & $18(32.7)$ & \\
\hline Female & $62(70.5)$ & $86(68.3)$ & $63(80.8)$ & $37(67.3)$ & \\
\hline Education & 87 & 125 & 78 & 55 & 0.377 \\
\hline Never/Primary & $25(28.7)$ & $42(33.6)$ & $20(25.6)$ & $12(21.8)$ & \\
\hline Secondary/University & $62(71.3)$ & $83(66.4)$ & $58(74.4)$ & $43(78.2)$ & \\
\hline Marital Status & 86 & 118 & 77 & 52 & 0.912 \\
\hline Marrieda/Living Together & $37(43.0)$ & $56(47.5)$ & $37(48.1)$ & $24(46.2)$ & \\
\hline Other & $49(57.0)$ & $62(52.5)$ & $40(51.9)$ & $28(53.8)$ & \\
\hline Health status & 88 & 126 & 78 & 55 & $<0.001^{*}$ \\
\hline Good & $63(71.6)$ & $112(88.9)$ & $68(87.2)$ & $52(94.5)$ & \\
\hline Altered & $25(28.4)$ & $14(11.1)$ & $10(12.8)$ & $3(5.5)$ & \\
\hline Alcohol intake & 88 & 126 & 78 & 55 & 0.206 \\
\hline No & $69(78.4)$ & $99(78.6)$ & $52(66.7)$ & $43(78.2)$ & \\
\hline Yes & $19(21.6)$ & $27(21.4)$ & $26(33.3)$ & $12(21.8)$ & \\
\hline Skin & 84 & 119 & 73 & 55 & 0.091 \\
\hline Normal & $62(73.8)$ & $97(81.5)$ & $60(82.2)$ & $50(90.9)$ & \\
\hline Lesion & $22(26.2)$ & $22(18.5)$ & $13(17.8)$ & $5(9.1)$ & \\
\hline Past history of TB & 88 & 126 & 78 & 55 & 0.079 \\
\hline Yes & $33(37.5)$ & $30(23.8)$ & $17(21.8)$ & $17(30.9)$ & \\
\hline No & $55(62.5)$ & $96(76.2)$ & $61(78.2)$ & $38(69.1)$ & \\
\hline Prophylactic cotrimoxazole & 88 & 126 & 78 & 55 & 0.531 \\
\hline Yes & 65 (73.9) & $95(75.4)$ & $52(66.7)$ & $38(69.1)$ & \\
\hline No & $23(26.1)$ & $31(24.6)$ & $26(33.3)$ & $17(30.9)$ & \\
\hline
\end{tabular}

*Chi-square test $(\alpha=0.05)$.

ancludes monogamous and polygamous marriage.

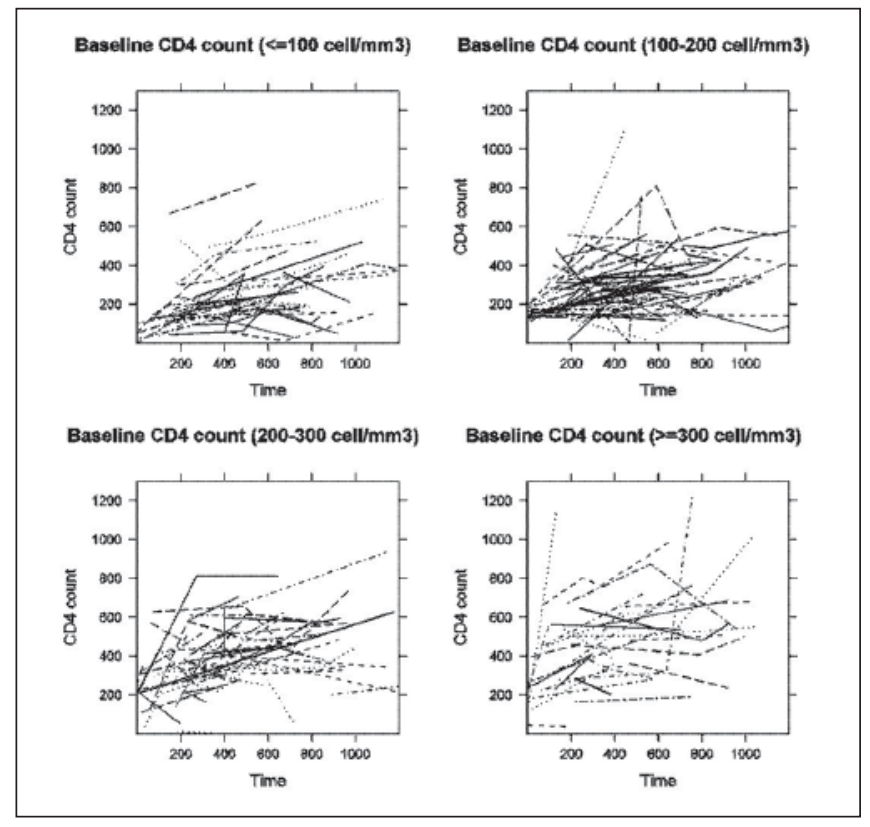

Figure 1: Observed CD4 count over time for different baseline CD4 count groups increase in the CD4 count across time and a possible quadratic trend, especially for the group of patients with CD4 count greater than 300 cells $/ \mathrm{mm}^{3}$. We also observed higher between-subject variations, especially in patients with baseline CD4 count greater than 300 cells $/ \mathrm{mm}^{3}$.

Mixed-effect regression models were used to evaluate the association between baseline variables and $\mathrm{CD} 4$ response over time. Baseline CD4 count was included in the model as a categorical variable, along with two-way interactions between baseline CD4 count group and time and baseline $\mathrm{CD} 4$ count group and the quadratic time term. Additional fixed effects included in the model were gender, health status, age group, country, marital status, education, alcohol intake, past history of $\mathrm{TB}$, skin condition, and use of prophylactic cotrimoxazole. The analysis of CD4 cell counts after ART initiation revealedthe following significant predictors of higher CD4 counts over time: gender, baseline CD4 count group, and the interaction between time and baseline CD4 count group and between 
the quadratic time term and baseline CD4 count group. As shown in Table 3, higher baseline CD4 cell counts predicted higher CD4 cell counts over time.

Figure 2 and 3 show the estimated mean CD4 counts over time by baseline CD4 count groups for males and females, respectively, accounting for the other variables in the model. Depending on the initial baseline CD4 count, increases in mean CD4 cell count ranged from 24-46 cells $/ \mathrm{mm}^{3}$ from 3to 6months after treatment initiation and from $80-117$ from 6to 18 months after treatment initiation. Females had higher CD4 counts at the initiation of treatment than males. Patients with higher CD4 count at ART initiation achieved a higher CD4 count in the following months.

\section{DISCUSSION}

Due to limited access to laboratory services such as CD4 count and viral load, many clinicians in Central Africa rely on WHO HIV clinical staging to inform treatment decisions, such as when to start and, if necessary, switch ART. This reality is reflected in our available data, given that 14,769 patients did not have CD4 counts and were thus excluded from this analysis. The limited access to laboratory services is exacerbated during public sector health care worker strikes, which further delay
Table 3: Parameter estimates and standard errors

\begin{tabular}{|c|c|c|c|}
\hline Variable & Estimate & $\begin{array}{l}\text { Standard } \\
\text { Error }\end{array}$ & p-value \\
\hline Baseline CD4 group & & & $<0.001^{*}$ \\
\hline$<=100$ & 227.55 & 40.94 & \\
\hline $101-200$ & 283.77 & 40.89 & \\
\hline $201-300$ & 379.70 & 42.05 & \\
\hline$>300$ & 483.28 & 46.11 & \\
\hline Baseline CD4 group*time & & & $<0.001^{*}$ \\
\hline$<=100$ & 0.21 & 0.059 & \\
\hline $101-200$ & 0.22 & 0.045 & \\
\hline $201-300$ & 0.23 & 0.054 & \\
\hline$>300$ & 0.29 & 0.065 & \\
\hline Baseline CD4 group*time ${ }^{2}$ & & & $0.019 *$ \\
\hline$<=100$ & -0.00010 & 0.000080 & \\
\hline $101-200$ & -0.00010 & 0.000086 & \\
\hline $201-300$ & -0.00023 & 0.000106 & \\
\hline$>300$ & -0.00040 & 0.000178 & \\
\hline Sex (Male) & -39.43 & 18.51 & $0.036^{*}$ \\
\hline Health (Good) & 46.12 & 24.02 & 0.058 \\
\hline Age group & & & 0.239 \\
\hline$<=34$ & -34.71 & 21.90 & \\
\hline $35-39$ & 1.91 & 23.97 & \\
\hline $40-44$ & -33.36 & 22.70 & \\
\hline Country (DRC/Burundi) & -9.72 & 22.97 & 0.673 \\
\hline Marital Status (Married) & 15.48 & 16.64 & 0.355 \\
\hline $\begin{array}{l}\text { Frequency of alcohol } \\
\text { intake(Never) }\end{array}$ & -0.99 & 18.86 & 0.958 \\
\hline Past history of TB & 1.28 & 18.80 & 0.946 \\
\hline Education (Never/primary) & -13.31 & 19.45 & 0.496 \\
\hline Skin condition(Normal) & 30.09 & 20.38 & 0.144 \\
\hline $\begin{array}{l}\text { Prophylactic } \\
\text { cotrimoxazole }\end{array}$ & -13.36 & 18.79 & 0.479 \\
\hline
\end{tabular}

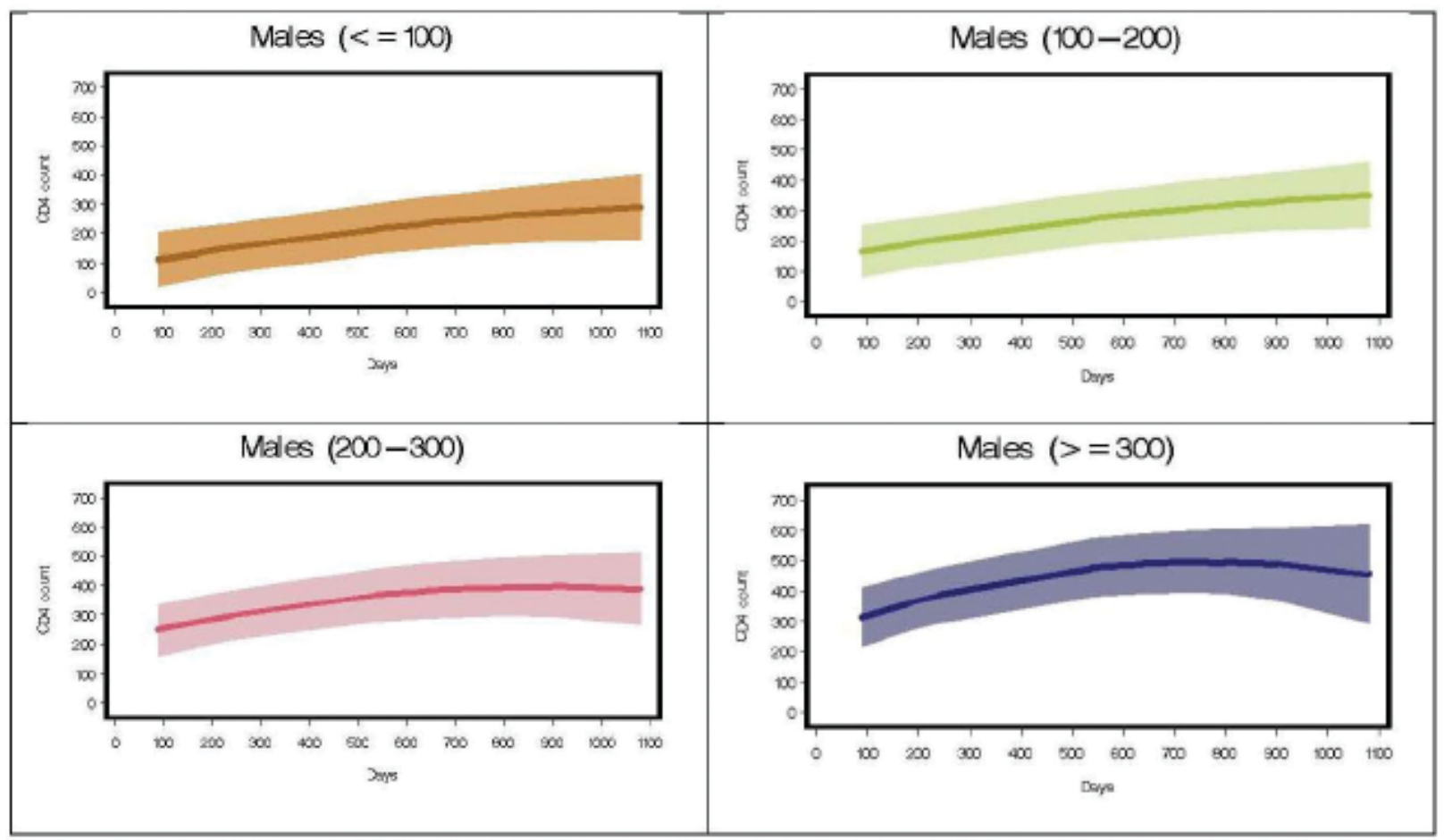

Figure 2: CD4 Progression over time for males after HIV treatment for 4 different baseline CD4 groups 


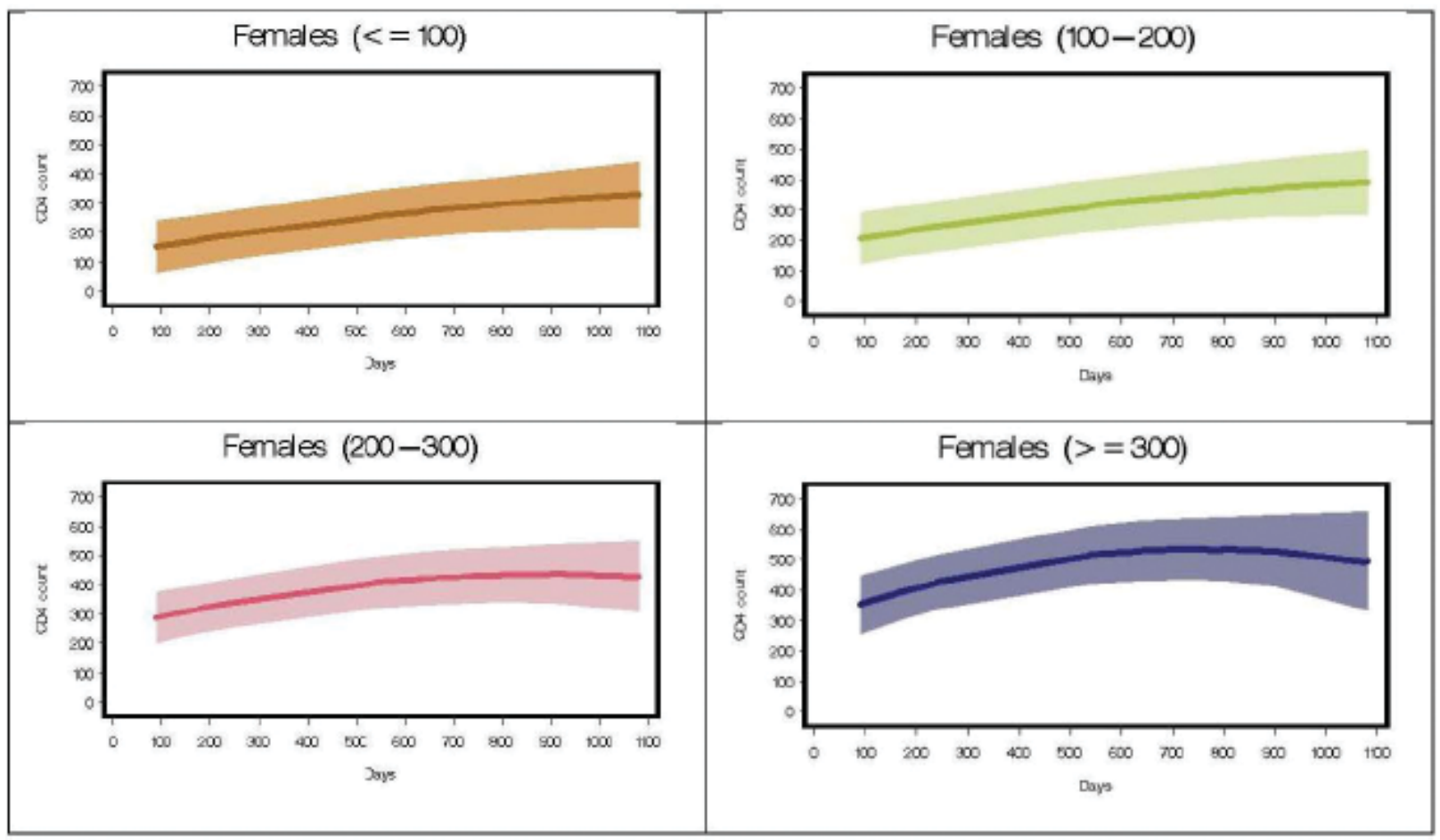

Figure 3: CD4 Progression over time for females after HIV treatment for 4 different baseline CD4 groups

laboratory results or makes them unavailable altogether. As demonstrated by the number of patients we excluded from this analysis due to lack of CD4 count, there is an urgent need for support to increase access to laboratory services in the central Africa region. The revised WHO [14]guidelines recommend ART for HIV-infected adults and adolescents who have CD4 cell counts less than or equal to 350 cells $/ \mathrm{mm}^{3}$. Increasing access to laboratory services in resource-limited settings should not be ignored while working to meet the increased demand for ART as the revised guidelines are put into practice.

Though this analysis included HIV-positive adults from DRC, Burundi, and Cameroon, $80 \%$ of patients eligible for this analysis were from DRC, 18\% were from Cameroon, and only 1\% was from Burundi. To address the possible effect that country may have had, we included country (DRC/Burundi and Cameroon) as a fixed effect in the mixed-effect regression models that we used to evaluate CD4 response over time. Country was not a predictor of higher CD4 counts over time, though additional data from Cameroon and Burundi would be helpful in making the results of this analysis more generalizable to the Central Africa region overall.

We found that higher baseline CD4 cell counts predicted higher CD4 cell counts over time among patients eligible for this analysis. This documentation of progression of CD4 counts over time by baseline CD4 count groups among eligible patientsprovides support for outreach efforts for early enrollment into HIV care, which is a precursor to ART initiation. Increases in mean CD4 cell count from 3 to 18 months after treatment initiation suggest that benefits of early initiation of ART are sustained though the duration of this follow-up time among these eligible patients. An increased sample size and longer-term follow-up is needed to determine whether higher baseline CD4 cell counts will continue to predict higher $\mathrm{CD} 4$ cell counts over time in this setting.

We observed that women had higher CD4 cell counts at the initiation of treatment than men. Women in this cohort tended to be younger than men, though few women were pregnant during our observation period. A total of 6 women were pregnant at the time of enrollment, and 19 additional women became pregnant during follow-up. No differences were observed between women and men for HIV clinical stage, health status, alcohol intake, past history of TB, prophylactic cotrimoxazole, and selfreported medication adherence. Numerous studies have assessed the differences in progression to AIDS and response to ART between men and women, and a recent review reveals conflicting support of these disparities [21]. Most studies in Nicastri's review reported no difference 
in viroimmunologic response to ART. However, a more recent study found that women in industrialized countries receiving ART progress more slowly to AIDS then men [18], and another states that women had higher likelihood of viral suppression than men [20]. Other evidence suggests that women respond more favorably to ART than men in terms of CD4 cell count [19]. Women comprise a larger proportion of incident HIV infections than do men [22], and this disparity is of particular importance for women of childbearing age, as prevention of maternal to child transmission (PMTCT) activities continue to be scaled-up in resource-limited settings. Additional inquiry is needed to determine why the women in this analysis tended to have higher CD4 count at ART initiation than men. Our findings suggest that early ART is beneficial for HIV-infected patients to maintain long-term high CD4 counts. A recent study clinical trial demonstrates clinical benefit of early ART in people with CD4 counts greater than 350 cells $/ \mathrm{mm}^{3}$, leading to a $96 \%$ reduction in HIV transmission to the HIV-uninfected partner [23].

Limitations of this study include a short follow-up period of 18 months, a heavy reliance on patients from DRC to form the sample for this analysis, and a modest sample size of 347 patients after excluding those with missing CD4 count. The proportion of the study cohort that has at least three follow-up visits is very small compare to the original sample may bias the results. Out of all enrolled patients in our clinics, $69 \%$ of them completed Secondary School orUniversity that is not a representative sample of the population. Despite these limitations, our results are noteworthy given the limited laboratory data available in the Central Africa region and the need to improve our understanding of CD4 progression over time in resource-limited settings. Further understanding may aid in the continued discussion of when to initiate ART and optimal timing for CD4 monitoring.

\section{ACKNOWLEDGEMENTS}

This studyfunded by the International epidemiologic Databases to Evaluate AIDS (IeDEA) grant (U01 AI069927) from the National Institute of Allergy and Infectious Diseases (NIAID), National Institutes of Health (NIH). The IeDEA-Central Africa collaboration acknowledges the contribution of local staff and patients in this project as well as staff at the IeDEA regional offices: Mr. Innocent Azinyue (Youndé, Cameroon), Dr. Modeste Kiumbu, Mr. Joseph Atibu, Mr. Christian Kondé, and Mr. Kashamuka Mwandagalirwa (Kinshasa, DRC).

\section{REFERENCES}

1. WHO. (2009a). Towards universal access: Scaling up priority HIV/ AIDS interventions in the health sector - Progress Report 2009. [electronic version]. Retrieved May 3, 2010 from http://www.who.int/hiv/pub/ tuapr_2009_en.pdf.

2. WHO. (2006). Antiretroviral therapy for HIV infection in adults and adolescents: Recommendations for a public health approach - 2006 revision [electronic version]. Retrieved May 3, 2010 from http://www.who.int/hiv/ pub/guidelines/artadultguidelines.pdf.

3. Ivers LC, Kendrick D, Doucette K. Efficacy of antiretroviral therapy programs in resource-poor settings: a meta-analysis of the published literature. Clinical Infectious Disease 2005:41(2), 217-224.

4. Braitstein P, Brinkhof MW, Dabis F, et al. Mortality of HIV-1-infected patients in the first year of antiretroviral therapy: Comparison between low-income and high-income countries. Lancet 2006:367, 817-824.

5. Tuboi SH, Brinkhof MW, Egger M, et al. Discordant responses to potent antiretroviral treatment in previously naïve HIV-1 infected adults initiating treatment in resource-constrained countries: the antiretroviral therapy in low-income countries (ART-LINC) collaboration. Journal of Acquired Immune Deficiency Syndrome 2007:45(1): 52-59.

6. Sabin CA, Phillips AN. Should HIV therapy be started at a CD4 cell count above $350 \mathrm{cell} / \mathrm{microl}$ in asymptomatic HIV-1 infected patients? Current Opinion of Infectious Disease 2009:22(2): 191-197.

7. Severe P, Pape J, Fitzgerald D, et al. A Randomized Clinical Trial of Early Versus Standard Antiretroviral Therapy for HIV-Infected Patients with a CD4 T Cell Count of 200-350 Cells/ml (CIPRAHT001). 49th Interscience Conference on Antimicrobial Agents and Chemotherapy; San Francisco, 2009; Abstract H-1230c.

8. Sterne JA, May M, Costagliola D, et al. Timing of initiation of antiretroviral therapy in AIDS-free HIV-1-infected patients: a collaborative analysis of 18 HIV cohort studies. Lancet 2009:373, 1352-1363.

9. Ford N, Calmy A, Hurst S. When to start antiretroviral therapy in resourcelimited settings: a human rights analysis. BMC International Health and Human Rights 2010:10, 6

10. Havlir DV, Getahun H, Sajournale I, et al. Opportunities and challenges for HIV care in overlapping HIV and TB epidemics. Journal of the American Medical Association 2008:300(4), 423-430.

11. Fox MP, Sajournale IM, Conradie F, et al. Initiating patients on antiretroviral therapy at CD4 cell counts above 200 cells/microl is associated with improved treatment outcomes in South Africa. AIDS 2010:24(13), 2041-2050

12. Badri M, Cleary S, Maartens $\mathrm{G}$, et al. When to initiate highly active antiretroviral therapy in sub-Saharan Africa? A South Africa cost-effectiveness study. Antiretroviral Therapy 2006:11(1): 63-72.

13. Walensky RP, Wolf LL, Wood R, et al. When to start antiretroviral therapy in resource-limited settings. Ajournalals of Internal Medicine 2009:151(3): 157-166.

14. WHO. (2009b). Rapid advice: Antiretroviral therapy for HIV infection in adults and adolescents-November 2009 [electronic version].Retrieved April 30, 2010 from http://www.who.int/hiv/pub/arv/rapid_advice_art.pdf.

15. Moore RD, Keruly JC. CD4+ cell count 6 years after commencement of highly active antiretroviral therapy in persons with sustained virologic suppression. Clinical Infectious Disease 2007:44(3), 441-446.

16 Kitahata M, Gange S, Abraham A, et al. Effect of early versus deferred antiretroviral therapy for HIV on survival. The New England Journal of Medicine 2009:360(18), 1815-1826.

17. DART Trial Team. Routine versus clinically driven laboratory monitoring of HIV antiretroviral therapy in Africa (DART): a randomized non-inferiority trial. Lancet 2010:375, 123-131.

18. Jarrin I, Geskus R, Bhaskaran K, et al. Gender differences in HIV progression to AIDS and death in industrialized countries: slower disease progression following HIV seroconversion in Women. American Journal of Epidemiology 2008:168, 532-540.

19. Rajasekaran S, Jeyaseelan L, Raja K, et al. Increase in CD4 cell counts between 2 and 3.5 years after initiation of antiretroviral therapy and determinants of CD4 progression in India. Journal of Post-graduate Medicine 2009:55, 261-266.

20. Kipp W, Alibhai A, Saunders LD, et al. Gender differences in antiretroviral treatment outcomes of HIV patients in rural Uganda. AIDS Care 2010:22(3): 271-278. 
21 Nicastri E, Leone S, Angeletti C, et al. Sex issues in HIV-1-infected persons during highly active antiretroviral therapy: a systematic review. Journal of Antimicrobial Chemotherapy 2007:60, 724-732.

22. UN (2009). United Nations Joint Programme on HIVIAIDS. 2009 AIDS Epidemic Updated. Retrieved May, 19.2010 from http://data.unaids.org/ pub/Report/2009/JC1700_Epi_Update_2009_en.pdf.
23. Cohen M. (2011), Treating HIV-infected People with Antiretrovirals Protects Partners from Infection. National Institute of Allergy and Infectious Diseases, Retrieved June, 28.2011 from http://www.niaid.nih.gov/news/ newsreleases/2011/Pages/HPTN052.aspx. 